\title{
The Curvature Effect
}

\author{
G. Corradi \& E. Munar
}

\section{Preprint published in:}

Corradi, G., \& Munar, E. (2020). The Curvature Effect. In M. Nadal \& O. Vartanian (Eds.), The Oxford Handbook of Empirical Aesthetics (Vol. 34, Issue 1, pp. 35-52). Oxford University Press. https://doi.org/10.1093/oxfordhb/9780198824350.013.24

\begin{abstract}
Preference for curved over sharp-angled contours is a well-known effect. However, it was quite unexplored during the $20^{\text {th }}$ century and only a few sporadic studies dealt with it. Nevertheless, there has been renewed interest in this topic over the last two decades. This interest has come from two perspectives, one related to the current experimental aesthetics and the other from different applied approaches: marketing, packaging, interior design, and security perception, among others. Quite a few studies have demonstrated the effect with different stimuli, conditions, and participants. However, a comprehensive understanding of this effect is still lacking. We present the salient issues of the current studies in order to provide a more complete picture of this phenomenon. The applied research line is a promising field to combine with research from experimental aesthetics. Finally, we indicate a few challenges that experimental research should address to achieve a unified framework for a better understanding of the curvature effect.
\end{abstract}

Keywords: Preference for curvature, contour, visual preference, curvature effect 
Contour is a fundamental basic visual attribute to properly shape an object's form. As Marr (1982) indicated, contour in a single two-dimensional image conveys unambiguous and often quite detailed information about the three-dimensional form. Shaping the object's form is a crucial visual process in order to recognize and appraise it. Thus, it is reasonable to think that different contours and lines -which make up contours- give rise to different feelings.

Even before the foundations of empirical aesthetics as an academic discipline were laid, Hogarth (1753), an English painter and writer, argued that curved lines are ornamental because they can vary in length and also in degrees of curvature, whereas straight lines vary only in length, and therefore are less ornamental. He added that the wavy line which he called "the line of beauty"- is "more productive of beauty", because it is composed of two contrasted curves and varies more insomuch as the hand makes a lively movement when drawing it with a pencil. It is probably the earliest study in which we can find a theoretical account of why curvature is a key factor in visual aesthetics (Bertamini \& Palumbo, 2015; Bertamini, Palumbo, Gheorghes, \& Galatsidas, 2016).

Quite a few studies have since shown that curved contours are preferred or involve more positive feelings than sharp-angled ones (Bar \& Neta, 2006; Gerardo GomezPuerto, Munar, \& Nadal, 2016; Leder \& Carbon, 2005). Preference for curvature has been shown with different kinds of stimuli: lines (Bertamini et al., 2016; Hevner, 1935; Lundholm, 1920; Poffenberger \& Barrows, 1924; Uher, 1991), typefaces (Kastl \& Child, 1968; Velasco, Woods, Hyndman, \& Spence, 2015), car interior designs (Leder \& Carbon, 2005), familiar objects (Bar \& Neta, 2006, 2007; G Gomez-Puerto et al., 
2017; Leder, Tinio, \& Bar, 2011; Munar, Gómez-Puerto, Call, \& Nadal, 2015), meaningless patterns (Bertamini et al., 2016; Cotter, Silvia, Bertamini, Palumbo, \& Vartanian, 2017; Palumbo \& Bertamini, 2016; Palumbo, Ruta, \& Bertamini, 2015; Quinn, Brown, \& Streppa, 1997; Silvia \& Barona, 2009; Velasco, Salgado-Montejo, et al., 2016), wrapped candies (Munroe, Munroe, \& Lansky, 1976), toys (Jadva, Hines, \& Golombok, 2010), furniture (Dazkir \& Read, 2012), interior designs (Banaei, Hatami, Yazdanfar, \& Gramann, 2017; Van Oel \& Van den Berkhof, 2013; Vartanian et al., 2013, 2017), product designs (Westerman et al., 2012), dancers (Aronoff, 2006; Aronoff, Woike, \& Hyman, 1992; Christensen, Pollick, Lambrechts, \& Gomila, 2016), interactive objects (Soranzo, Petrelli, Ciolfi, \& Reidy, 2018), and even haptic forms (Jakesch \& Carbon, 2011). Moreover, the effect has been shown in adults -most of the aforementioned studies-, newborns (Amir, Biederman, \& Hayworth, 2011; Fantz, 1961; Fantz \& Miranda, 1975; Quinn et al., 1997; Ruff \& Birch, 1974), infants (Hopkins, Kagan, Brachfeld, Hans, \& Linn, 1976; Jadva et al., 2010; Munroe et al., 1976), western and non-western participants (G. Gómez-Puerto et al., 2017), and even in great apes (Munar et al., 2015) and chicks (Fantz, 1961; Schneirla, 1965). Nowadays, we can state that the "preference for curvature" or the "smooth curvature effect" or simply the "curvature effect" is a well-established phenomenon.

\section{Sporadic studies over the $20^{\text {th }}$ century}

The earliest experimental research about preference for curvature that we found was described in Stratton $(1902,1906)$. He related the pleasure derived from the observation of curved lines to the concurrent movements of the extra-ocular muscles. He 
hypothesized that eye movements required to follow sharp lines must be more abrupt and, consequently, less pleasant than those required to follow curved lines. Stratton recorded gaze patterns while participants viewed different kinds of curved and sharpangled stimuli using a rudimentary eye-tracking device. He noted that participants' eyes moved jerkily and similarly in relation to both curved and sharp-angled stimuli. However, despite the fact that his proposal was unsupported based on his own observations, his seminal work included interesting reflections on the two issues that have become central in the current research (Gerardo Gomez-Puerto et al., 2016). The first one was about the mechanism underlying preference for curvature. He believed that curves provide observers with a continuous flow of information that is easy to process. However, he noticed that experience, environment, and cultural cues might influence the appraisal of lines. The second issue was about the functional significance of this preference. Most movements in nature are curvilinear, which makes us perceive the curved lines as an indication of a functional, normal behavior. On the other hand, angled lines convey a stronger sense of power.

Despite Stratton's inspiring experimental beginning to study the curvature effect, there were very few empirical attempts to delve into this issue during the $20^{\text {th }}$ century. In the first half of the century, some studies related specific lines to specific feelings (Hevner, 1935; Lundholm, 1920; Poffenberger \& Barrows, 1924). Participants received either (1) a series of words or (2) a series of lines and they had either (1) to draw lines matching the words, or (2) to match some feelings to the lines. Some of the results related curved lines to sad, quiet, lazy, merry, dead, playful, weak, gentle, serious, graceful, and serene; and in turn sharp lines to agitating, furious, robust, vigorous, and hard. The patterns of response were quite consistent and uniform for most participants. 
In an applied approach, Kastl and Child (1968) supported the notion that distinct feelings conveyed by isolated lines remain relevant when the stimuli are more complex. Using typefaces, they found that, in general, moods such as sprightly, sparkling, dreamy, calm, and soaring tend to be matched to the curved type, whereas moods such as dignified are matched to the angular type. Nevertheless, it should be noted that their findings indicated that angular types appeared to be sadder, contravening those of Lundholm (1920).

Valentine (1950) suggested that the curvature effect might be connected with the appeal of curves in the human form. McElroy (1954) indicated that the form most strongly appreciated as beautiful is the opposite sex, and much of the pleasure in works of art may be due to their appeal to sex-related emotions. He considered the role played by symbolism in mental life and alleged that sex differences must also exist regarding symbolic representations of male and female forms. Male perception of any suitable symbolic representation of the female form might be accompanied by a surplus of pleasurable affect, with a converse process applying to female perception of any suitable representation of the male form. As simple curved figures may represent female forms and pointed forms are typical male symbols (Franck \& Rosen, 1949; Krout, 1950), McElroy constructed a twelve-item aesthetics test to measure sex differences in aesthetic perception. Each item consisted of a pair of line drawings, one selected on account of its curved features (feminine) and the other selected because of its pointed properties (masculine). From a psychoanalytic hypothesis, he predicted that "surplus of affect" would exist and would be distributed in opposite directions for male and female groups. The results partially supported his prediction and showed that this sex 
difference increased with age. In this sense, McElroy interpreted this as implying that the difference was culturally delimited.

In the same line, Jahoda (1956) used McElroy's test with 858 boys and girls from Ghana. The results showed reduced but significant sex differences. He argued that the psychoanalytic hypothesis was supported and that the smaller differences in Ghana's group were due to a different cultural situation where there was less sexual repression. In contrast, Munroe (1976) asked 175 children to select between two pieces of wrapped candies, one spherical and the other cube-shaped. Participants from both sexes chose the spherical candy more frequently. However, girls chose it (83\%) significantly more than boys $(57 \%)$. Since then, current studies have not found any sex differences in the curvature effect (Bar \& Neta, 2007; Jadva et al., 2010; Palumbo et al., 2015).

In a subliminal perception study, Guthrie and Wiener (1966) presented drawings to participants who had to make judgments about a man in the drawing, using a list of several trait-dimensions (pleasant-unpleasant, helpful-harmful, ...). They created two different versions of each image with sharp and smooth contours. The results showed that it was the overall sharpness, and not the presence of a gun, that determined whether the image was perceived as threatening and negative.

In the seventies, it was shown that 13-week-old infants (Ruff \& Birch, 1974) and even 1-week-old neonates (Fantz \& Miranda, 1975) fixated longer on curved contour forms than on straight or sharp-angled contours, respectively. The view of the infants was limited to two stimulus patterns. Hopkins et al.'s (1976) results also implied that curvature appeared to have a special attention-recruiting quality for 10-month-old 
infants. Once infants were habituated, pressing a lever, to a straight or curved line, the experimenter changed the type of line that appeared when the infant pressed the lever and the dishabituation process were measured by means of several variables. Results showed that sustained reinforced responding and visual fixation were greater when dishabituation involved a change from straight to curved rather than a change from curved to straight form. This greater responsivity, when the change is from a straight to a curved segment than the opposite direction, seems due to a greater interest and excitement for curved lines for the infant.

Uher (1991) presented stimuli with zigzags and wavy lines to 1,100 participants of various ages, genders, and occupations from Central Europe. They rated the emotional qualities on 24 semantic differential scales. Her results showed significant associations with antagonistic characteristics for the zigzag and with affiliative ones for the wavy lines. Interestingly, the author, from an ethological perspective, concluded that the zigzag motifs were likely to represent entoptic phenomena, that is, visual effects whose source is within the eye itself.

Aronoff et al. (1992) hypothesized that geometric patterns permit observers to recognize the meaning of threat or warmth. They used videotapes of classical ballet performances in Study 1, and lines and elliptical forms in Study 2. In Study 1, they analyzed three main visual configurations in human movement in a ballet performance: body display, arm display, and movement path. The body display was categorized in diagonal and round movements. The arm display and the movement path were categorized in angled, straight, and round movements. Roundness in the visual displays was strongly associated with warm characters, whereas the reverse visual display was strongly 
associated with threatening characters. They concluded that it was the abstract geometric form made in the body's presentation that provided the meaning. Christensen et al. (2016) replicated these results. In Study 2, Aronoff et al. (1992) carried out three experiments with diagonal lines and elliptical forms that were rated on a semantic differential scale with 12 adjective pairs. Again, roundedness produced a very large effect on the evaluation dimension, with a more rounded form seen as being better. A V-shaped figure -with a downward vertex- was perceived to be significantly worse than all the other diagonally shaped forms. The authors concluded that there is some neural mechanism that may respond to rounded visual forms with the meaning of warmth and may respond to diagonal, linear, and angular visual forms with the meaning of threat, whether the actual physical object that manifests the shape is a face, a line, a schematic drawing, or a body movement (Aronoff, 2006).

Quinn et al. (1997) showed that Gestalt organizational effects and preference for curvature are both involved in the initial parsing and subsequent organization of complex visual patterns. Using a familiarization-novelty preference procedure, the authors found that 3- and 4-month-old infants segregated the contours of two intersecting visual forms, and that they did so relying on the Gestalt principle of good continuation. Moreover, they argued that spontaneous preference for curvature facilitated the Gestalt organization of complex configurations into coherent forms.

\section{The $21^{\text {st }}$ century}


Most of the $20^{\text {th }}$ century contributions to the study of the curvature effect were incidental and sporadic. They did not construct a body of knowledge about the psychological and biological functions of the phenomenon. In the $21^{\text {st }}$ century, something in this regard has changed. In the first decade of the current century, several systematic studies focused on this effect (Bar \& Neta, 2006, 2007; Leder \& Carbon, 2005; Silvia \& Barona, 2009; Zhang, Feick, \& Price, 2006) and helped to increase interest about preference for curvature. Currently, we can find quite a few studies that have brought into focus the effect and have contributed to a better understanding of the phenomenon. However, these studies do not comprise a unified set that shares a common viewpoint. Actually, they make use of different frameworks. For example, some of them come from the applied perspective (Carbon, 2010; Dazkir \& Read, 2012; Leder, Carbon, \& Ripsas, 2006; Zhang et al., 2006) whereas others from a more basic, experimental standpoint (Bar \& Neta, 2006, 2007; Leder et al., 2011; Silvia \& Barona, 2009; Vartanian et al., 2013). Some of the contributions are unconnected, and we cannot yet offer a cohesive account that encapsulates the available evidence. Nonetheless, we are going to present the salient issues of the current studies in order to provide a constructive development.

An evolved or a learnt preference?

According to Carbon (2010), it is questionable whether the curvature effect can be demonstrated in all domains and for all times, especially when we take artificial, human-made objects into account. Leder and Carbon (2005) and Carbon (2010) relied on familiarity, mere exposure, and innovativeness to explain results from their studies on car design. When curved interiors were preferred to straight ones, the authors argued that straight lines were innovative and, therefore, the mere exposure effect could be held 
accountable for effects of preference for straightness, angularity, or curvature in different cases. To further explore this possibility, Carbon (2010) showed participants images of cars representing different epochs and styles. He indicated that curved car exteriors were only preferred when the design itself belonged to an epoch in which the trend was to build a more curved chassis. He claimed that preference for curvature was not static and uniform, but instead was under the influence of the aesthetic Zeitgeist of a given time. This reasoning supports a learnt curvature effect as well as Zhang et al.'s (2006) findings could do. According to them, countries high on individualism tend to use more angular brand logos than countries high on collectivism.

On another hand, before any experimental study about preference for curvature had been performed, Allen (1877) claimed a biological origin of some aesthetic features, including the curvature effect. According to this line of reasoning, Uher (1991) linked the use of zigzag motifs among different cultures to ancient environmental pressures. Her evidence supported the influence of our biological heritage in the use of zigzag motifs, an influence that, nonetheless, was susceptible to cultural modulation. There are some other results that support the idea of an evolutionary origin of the curvature effect. Fantz and Miranda (1975) showed that 1-week-old infants tended to look longer at curved stimuli than at sharp ones. Moreover, findings from our group revealed that preference for curvature is also present in non-Western cultures (Gerardo Gómez-Puerto et al., 2017; Munar, Gómez-Puerto, \& Gomila, 2014) and even among non-human primates (Munar et al., 2015), using in both cases a particular approach-avoidance paradigm.

The approach-avoidance paradigm, due to its relationship with the evolutionary perspective and the embodied mind framework, deserves a separate mention in this section. It brings experimental aesthetics closer to the most basic and primary 
behaviors, while avoiding, as much as possible, complicated cognitive interpretations both by participants and researchers (Elliot \& Covington, 2001; Munar et al., 2014). Some studies using approach-avoidance tasks found the curvature effect (Bertamini et al., 2016; Cotter et al., 2017; Gerardo Gómez-Puerto et al., 2017; Munar et al., 2015; Palumbo et al., 2015), whereas others have not (Vartanian et al., 2013; Velasco, Salgado-Montejo, et al., 2016). However, they used different tasks: Bertamini et al. (2016) and Palumbo et al. (2015) used the manikin task; Munar et al. (2015) and Gómez-Puerto et al. (2017) used a pair-comparison task with an approach simulation after response; and Velasco et al. (2016) asked participants to categorize shapes in terms of approach/avoidance words. Conversely, Vartanian et al. $(2013,2017)$ used the same task in both studies in which participants rated interior design images on a dichotomous willingness-to-enter scale (enter versus exit), obtaining opposing results. They did not find a curvature effect in the former but they did in the latter. The authors indicated methodological considerations to account for the difference in results. The response window in the former study was limited to three seconds, whereas there was no time limit in the latter study. It is possible that longer viewing time triggers top-down processes that might alter the initial, rapid response to contour. The direction of this alteration would vary as a function of the task under consideration (i.e., beauty judgment vs. approach-avoidance decisions). In addition, the two studies differed in the number of stimuli, as well as the settings in which the data were collected. Perhaps most importantly, whereas one study involved naïve subjects, the other involved experts in the form of architects and designers. It is possible that expertise alters one's response to curvature in the context of approach-avoidance decisions. Further research is needed to clear up these aspects.

Overall, there is a certain amount of evidence supporting a possible evolutionary origin 
for the curvature effect, and several hypotheses that could explain it. One of them has been the threat hypothesis (Bar \& Neta, 2006, 2007), which we will expound later. Another one is based on the neotenic features that tend to result in salient curved configurations, such as a rounded face or large rounded eyes, and seem to have been favored by sexual selection (Bertamini et al., 2016).

\section{A sensorimotor or an appraisal mechanism?}

Some explanations of the curvature effect are based on specific sensorimotor systems, and others on appraisal processes (Gerardo Gomez-Puerto et al., 2016). The sensorimotor explanation comes from the way in which curved stimuli directly interact with specific activation of the sensorimotor mechanisms (Amir et al., 2011; Fantz \& Miranda, 1975; Ruff \& Birch, 1974; Stratton, 1902, 1906). This research line is partly related to the processing fluency theory of aesthetic pleasure (Reber, Wurtz, \& Zimmermann, 2004). Accordingly, fluent processing of an object leads to positive aesthetic responses. From this perspective, preference for curvature is greater than preference for angularity because curvature facilitates processing fluency. Some studies have reported that participants carry out different tasks faster using stimuli with

curvilinear features than rectilinear ones (Álvarez, Blanco, \& Leirós, 2002; LoBue, 2014; Quinn et al., 1997; Ruta, Palumbo, \& Bertamini, 2014; Treisman \& Gelade, 1980; Wolfe, Yee, \& Friedman-Hill, 1992). However, Bar and Neta $(2006,2007)$ found no difference in the time it took participants to rate curved and sharp stimuli, even when curved ones were preferred. So far, only one preference-for-curvature study has reported significant differences between curved and sharp stimuli in response time. Experiment 1 in Palumbo and Bertamini (2016) showed that responses for the curved 
shapes were faster compared to responses for the sharp-angled shapes. This led to thinking that people perform general tasks faster with curvilinear than sharp-angled stimuli but this is not usually the case in tasks related to preference.

Amir et al. (2011) found that adults and infants looked first, and adults looked longer, at curved and non-parallel geons than at straight and parallel ones. According to them, human saccades tend to maximize the rate of information acquisition. First fixations tend to go to salient locations (Itti \& Koch, 2001) and to locations that present greater uncertainty or a maximum amount of local information (Renninger, Verghese, \& Coughlan, 2007). Amir et al. (2011) also found that curved geons produced great BOLD (Blood Oxygen Level Dependence) activity in human shape-selective cortex, that is, lateral occipital cortex and posterior fusiform gyrus. The importance of curvature in occipitotemporal cortex is emphasized by the existence of a distributed network of cortical patches that respond maximally to curved stimuli (Yue, Pourladian, Tootell, \& Ungerleider, 2014). Curved stimuli activate most V4 sites, and concave curvature relative to fixation point- activates many posterior inferotemporal cortex sites (Ponce, Hartmann, \& Livingstone, 2017).

Other explanations for the curvature effect have focused on appraisal mechanisms (Bar \& Neta, 2006, 2007; Leder et al., 2011; Vartanian et al., 2013). These appraisal approaches are motivated by early findings about the emotional evaluation of straight, sharp, and curved lines (Lundholm, 1920; Poffenberger \& Barrows, 1924), and abstract shapes (Hevner, 1935). Guthrie and Wiener's (1966) findings -which showed that the overall sharpness of a drawing determined that the image was perceived as threatening and negative-, and Kastl and Child's (1968) findings -about the emotional meaning of different typographies- lent additional support to this approach. This research line is based on implicit and explicit appraisal processes and the way in which they impact 
aesthetic experience. These authors tend to agree that curvature is imbued with nonrepresentational semantic meaning and relate it to emotional processes. According to Vartanian et al. (2013), the effect is probably driven by pleasantness. Their results showed that judging the beauty of curvilinear spaces was associated with an increase in anterior cingulate cortex (ACC) activity. They indicated the contribution of ACC to reward and emotional and affective processing. Using a mobile EEG and head-mounted virtual reality, Banaei et al. (2017) also found stronger theta synchronization in the ACC with curved forms.

Implicit measures might reveal more information about the semantic/affective processes underlying preference as compared to explicit evaluations. Palumbo et al. (2015) tested implicit associations between shapes' contour line (curved vs. sharp-angled) and attributes: valence, danger, and gender. They used an Implicit Association Test (IAT) with irregular polygons that allowed the control of familiarity and semantic processes, which typically influence liking evaluations. Curved shapes were implicitly associated with safe and positive words and with female names. In contrast, angular shapes were associated with danger and negative words and with male names. The test revealed affective processes underlying the curvature effect, which might not be accessible with explicit evaluations. The authors suggested that preference for curved contours might be triggered by an aesthetic quality contained in the curved contour itself, but it is also mediated by what curves can evoke in terms of affective representations. They indicated that preference for curves might be boosted by positive feedback from second-order associative processes.

The threat hypothesis 
Aiken (1998) argued that preference for curvature is actually motivated by fear induced by sharp lines. Such fear had served an adaptive function in our past to help us rapidly detect and avoid possible threats. The author stated that primitive emotions, which originated initially as a response to environmental pressures, have been repurposed, giving rise to the aesthetic experience.

Bar and Neta $(2006,2007)$ tested the hypothesis that curved stimuli are preferred because sharp contours evoke a sense of threat. They confirmed the curvature effect with images of familiar objects and meaningless patterns. Participants were asked to make a like/dislike choice about stimuli varying in their contour: curved, sharp, or mixed. Curved stimuli were liked more than mixed and sharp ones (Bar \& Neta, 2006). In another experiment, participants had to respond "threatening" or "non-threatening" to each stimulus. Sharp-angled objects and patterns were rated as significantly more threatening than curved ones. The authors interpreted their findings as being consistent with the proposal that objects may be perceived as threatening based on the nature of their contour.

Moreover, in an fMRI study, Bar and Neta (2007) observed a bilateral increase in amygdala activity when participants were presented with sharp stimuli. The authors expected this result, as amygdala activity is related to threat perception. Grassini et al.'s (2019) results supported this line of argument, but with other stimuli: the curved ones. In Experiment 1, they compared snakes, ropes, and other items, and the results showed that both snake and rope images elicited enhanced P1 and N1 event-related potential components as well as early posterior negativity (EPN). In Experiment 2, they studied whether nonthreatening curvilinear images (i.e., ropes) still elicited the enhanced 
electrophysiological responses when snake images were not presented as stimuli, and therefore, the context did not provoke top-down attention to curvilinear shapes. Rope images still evoked an enhanced EPN -but not P1 and N1. However, this effect was smaller than in Experiment 1, in which snake images were present. According to the authors, a stronger EPN response can be considered an index of reflexive attention and increased activity at the level of visual cortex, and might be the result of the influence of the amygdala activation on the visual areas. The authors suggested that the results might be due to a high-level, top-down attentional effect, such that the participants voluntarily directed attention toward curvilinear shapes when snakes were present as stimuli. Bottom-up attentional processes might also be involved, because the participants may have been automatically more aroused when snake images were present in the experiment.

As Bar and Neta $(2006,2007)$ used objects whose semantic meaning was emotionally neutral, Leder et al. (2011) further explored the contour effect with objects of negative valence. They hypothesized that if the perception of threat produced by an object was related to preference, then the negative valence could override other positive cues such as the curvature of its contour. Thus, they presented new stimuli depicting images of real objects that had been manipulated to create a curved and a sharp contoured version of each object. These objects were selected according to their emotional valence: positive or negative. They digitally manipulated one version of the object -curved or sharp - to create the other new version -sharp or curved. As predicted, curved stimuli were preferred to sharp ones, but only when the objects had a positive or neutral valence. They argued that this showed how threat and preference are interconnected, 
and that semantic evaluation takes precedence over the evaluation of contour, overriding the effects that curvature might have in preference.

However, some findings have questioned the threat hypothesis. In an fMRI study with images of interior architectural spaces, Vartanian et al. (2013) found no increase in amygdala activity when viewing rooms with sharp-angled contours compared to rooms with curved contours, which were preferred to the former. The authors suggested that sharp cues in buildings could have lost their threatening nature through learning and exposure.

Bertamini et al. (2016) and Palumbo et al. (2015) showed that angles are not necessary to generate preference for curvature. Bertamini et al. (2016) (Experiment 2) tested whether the effect was modulated by distance. If angular shapes were associated with threat, then the effect might be stronger when they were presented within peripersonal space. In the experiment, the shapes appeared at different perceived distances. The task was to rate preference for each shape after judging whether the object was within reach. According to the threat hypothesis, the effect should be stronger when shapes were presented near the observer, because any threat in near space is more noticeable than a threat farther away. However, the results showed that the curvature effect was not modulated by perceived distance. In Experiment 3, they used lines seen through an aperture so that there was no closed shape to form an object. There were straight lines with no angles, lines with an angle, and parabolas. If curvature was pleasant in itself, it should be preferred to straight lines, even in the absence of angles. The results revealed that curvature was preferred over angularity even for simple lines. Moreover, there was no difference in preference for angular and straight lines. In Experiment 4, they used a 
manikin task in which participants had to move a stick figure closer or farther, as instructed, from a series of curved or sharp polygons. Participants moved the manikin faster when presented with sharp polygons, independently of whether they brought it closer or moved it farther away. But when presented with curved polygons, they reacted faster only when they brought it closer to the stimuli. This is not the behavior that would be expected if sharp angles were perceived as threatening. Using the same task, Palumbo et al. (2015) found the same tendency with polygons with more and less pronounced vertices. The authors concluded that, in these cases, preference is due to the intrinsic characteristics of curvature and not to a rejection of sharp contours. However, they also indicated that the two proposals are not necessarily contradictory but instead, in some cases, might be complementary.

Soranzo et al. (2018) showed that sharp-angled objects displaying behaviors were preferred over quiescent objects and that the difference between preference for curved contours against sharp contours actually decreased when the object displayed behaviors. It is reasonable to believe that a potentially threatening motionless object would be more threatening if it were to display a behavior. Particularly, if an angularly shaped object exhibits behavior, this should be considered more threatening than a similar object that is quiescent. This finding also supports the hypothesis that the curvature effect is a genuine preference for curvature, rather than an avoidance of sharp-angled objects.

Beyond visual contour

Related to an evolutionary perspective, the idea that the curvature effect could be 
present in other sensory modalities has been widely expressed but little empirical evidence has demonstrated it. Jakesh and Carbon (2011) argued that the haptic sense should be less affected by cultural and Zeitgeist aspects and thus, should be more directly affected by evolutionary-based influences. Consequently, they used 3D plotted artificial stimuli with two types of contours (curved and sharp-angled) and two complexity levels (low and high). They used two types of responses: a like/dislike response and a 7-point scale. Curved stimuli were significantly rated as preferable in both types of responses.

Soranzo et al. (2018) reported the curvature effect with 3D objects that were both looked at -sight- and manipulated -touch. It seems therefore that the effect is not limited to pictorial representations and to visual processing, but it might be a general feature that extends to $3 \mathrm{D}$ objects and influences experience in more perceptual domains, such as touch.

Besides visual and tactile contour, preference for curvature has also been revealed in another perceptual attribute, that is, body movement. As mentioned previously, warmth has been related to curved body movements, curved arm movements, and curved movement paths in ballet performances (Aronoff, 2006; Aronoff et al., 1992; Christensen et al., 2016).

On another hand, there are several findings that showed a significant relation between sweet taste and curved shapes (Blazhenkova \& Kumar, 2018; Salgado-Montejo et al., 2015; Velasco, Woods, Petit, Cheok, \& Spence, 2016). If sweet tastes are considered to be preferred to other tastes, an indirect relationship between curved shapes and 
preferred taste can be established. However, empirical support for this association is required.

More studies are needed to confirm or reject the curvature effect in non-visual modalities, especially in audition. In any case, the previous findings are indicating a possible similar effect in other modalities. If the effect were confirmed in other sensory modalities, then we should wonder about a possible common origin among modalities or specific sources for each modality. These visual and haptic preferences might have a common origin, with one perhaps being a consequence of the other, a matter that should be solved empirically. Following Dewey's (1922) principle of continuity, it would also be interesting to study whether these preferences could be recruited for higher cognitive consequences, as some instances in language could show: "well rounded," "sharp practice," "come round," "a short, sharp shock," "the sharp end," and "sharp" as "not quite honest" (Munar et al., 2014).

\section{Individual and contextual differences}

As the curvature effect has been firmly demonstrated across age groups, cultures, and species, a logical step is to explore different moderators such as personal and contextual features to shed light on its nature and mark the boundaries of the effect. In this line, several studies have focused on individual differences regarding the curvature effect (Cotter et al., 2017; Silvia \& Barona, 2009; Vartanian et al., 2017; Zhang et al., 2006).

Using brand logos as stimuli, Zhang et al. (2006) found that people with an activated inter-independent self-construal (i.e., emphasizing compromise and collectivism) perceived curved shapes as more attractive than people with an activated independent self-construal (i.e., emphasizing confrontation and individualism). Moreover, they 
showed that countries high on collectivism (Hong Kong, Korea, and Japan) tended to use less angular logos than countries high on individualism (United States, Canada, United Kingdom, and Germany). Tzeng et al. (1990) also found intercultural differences in the perception of what they called the "rounded factor" despite some similarities between cultures. They used 21 semantic differential scales, 10 graphic stimuli, and three groups of participants from Japan, Mexico, and Columbia. The "rounded factor" was the factor that accounted for more variance in a principal component analysis based on the data from the semantic differential scale. The Japanese sample liked roundness more than Mexican and Columbian samples.

However, Gómez-Puerto et al. (2017) investigated whether preference for curved contours was common across different cultures. They tested preference for curvature in two small-scale societies relatively uninfluenced by Western culture (Oaxaca, Mexico, and Bawku, Ghana) and a university sample from a Western country (Majorca, Spain). Participants performed a two-alternative, forced-choice task, in which they had to choose between curved and sharp-angled versions of the same real objects. The results showed that participants in all of the three countries chose the curved-contour version significantly more often than the sharp-angled one. Moreover, preference did not differ significantly among the three samples. The authors concluded that the curvature effect seems to be common across cultures and the conjecture that it is a constituent of a natural propensity for aesthetics.

On another hand, some findings indicate that artistic expertise interacts with the curvature effect (Cotter et al., 2017; Silvia \& Barona, 2009; Vartanian et al., 2017). This interaction seems to depend on the type of stimuli. With asymmetrical complex patterns 
(Silvia \& Barona, 2009) or irregular polygons (Cotter et al., 2017), participants higher in artistic expertise showed a greater curvature effect. Conversely, with arrays of simple objects -circles and hexagons-, either there was no difference between experts and novices (Cotter et al., 2017) or the novices presented the effect and the experts did not (Silvia \& Barona, 2009). Circles and hexagons are familiar objects, but the irregular stimuli used by Silvia and Barona (2009) and Cotter et al. (2017) were novel. As a possible explanation of the results, the authors indicated that people possess linguistic labels for circles and hexagons with a set of established semantic associations. It is likely that these stimuli already possess valence, which can take precedence over curvature (Leder et al., 2011).

Vartanian et al. (2017) studied the effect of interior space contour -curvilinear versus rectilinear- with architects, designers, and non-experts. Participants rated photographs of architectural spaces -half on a dichotomous beauty scale and half on a dichotomous willingness to enter/exit scale -to indicate whether it was a space they would like to enter or leave. With the beauty scale, experts showed preference for curvature but nonexperts did not. With the enter-exit scale, non-experts presented the curvature effect but experts did not. The non-experts' results were the opposite of those of Vartanian et al. (2013). They showed the curvature effect with the beauty scale and not with the enterexit scale. Aside from the fact that some of the photographs were the same in the two studies, there were some differences between the two procedures. The authors focused on the deduction that contour might be a more salient variable to architects and designers when assessing beauty. Another possibility is that contour could be a function of negative associations toward rectilinear contours, honed through professional practice and training. In relation to the enter/exit results, they suggested that experts 
might be trained to view images of architectural spaces dispassionately and be less subject to implicit behavioral biases rendered by their visual features. On another hand, the functionality or usability of a space becomes the relevant frame for choice for nonexperts.

Another trait that seems to moderate the curvature effect is openness to experience. Cotter et al. (2017) measured this trait with the HEXACO Inventory, the Neo-FiveFactor Inventory, and the Big Five Aspects Scale. Participants scoring higher on these scales showed greater curvature effect in irregular polygons, but not in arrays of circles and hexagons.

Type of stimuli and presentation time

Besides personal and situational features, there are some other moderators of the effect that come from the stimuli. As Cotter et al. (2017) found, irregular polygons and arrays of circles and hexagons interact differentially with expertise. Regarding the familiarity of the stimuli, Bar and Neta (2006) reported a slightly larger effect size using meaningful images than meaningless patterns. However, Leder et al. (2011) reported a slightly larger effect size with abstract patterns than with real object images. Both studies used the same presentation time $(84 \mathrm{~ms})$, the same like-dislike task, and the same stimuli. Westerman et al. (2012) found similar effect sizes in meaningless patterns and real objects, using designs of packaging and until-response conditions.

In Corradi et al. (2018) we found similar effect sizes with real objects and meaningless patterns presented for short-medium times $(84,150,300 \mathrm{~ms})$, but different effect sizes for long times. The effect faded in the presentation time-until response with images of 
real objects as in Munar et al. (2015), whereas it increased with meaningless patterns. We interpreted this as implying that the processing of the two types of stimuli must be different. We hypothesized that long presentations give rise to an increasing contribution of semantic content that leads to preference based on such content instead of on curvature. When shapes have no meaning, the curvature effect is preserved or even heightened in long presentations. According to our results, this might come from the intensified weight of the curvature in the discrimination and decision processes.

Palumbo and Bertamini (2016) also varied the presentation time using the same meaningless patterns as we did: $120 \mathrm{~ms}$ in Experiment 1 and until-response in Experiments 2 and 3. The effect size was slightly higher in Experiment 1 than in Experiments 2 and 3. However, the difference between their results and ours could come from several sources: Instructions, type of presentation, and kind of response. Palumbo and Bertamini's instructions were about liking in Experiments 1 and 2, and about attractiveness in Experiment 3. Our instructions were about selecting one of the two stimuli presented at once. On another hand, they used a like/dislike response in Experiment 1 and a rating scale in Experiments 2 and 3. They also manipulated the number of vertices and concavities. In this line, they concluded that parametric manipulations of complexity produce small but significant effects on preference. In particular, more concavities within curved shapes introduce more changes in curvature -and higher curvature magnitude-, in turn, decreasing liking.

\section{Applied science}

In the last decade, quite a few research lines have focused on understanding how object 
contours influence people to design more adaptive interactions between objects, or arrangements, and individuals. Some of them have tried to link basic scientific findings with applied aims, and others have had more direct business purposes. Because of the applied orientation, some of the tasks in these studies have not been about direct preference but about feelings that curvature can convey. Research from domains such as advertising, marketing, packaging, interior design, and planning of urban and recreational environments has taken interest in the curvature effect. We distinguish three types of applied studies according to their focus: (a) item forms (Ghoshal, Boarwright, \& Malika, 2016; Hareli, David, Lev-Yadun, \& Katzir, 2016; Hůla \& Flegr, 2016; Soranzo et al., 2018); (b) product packaging, logos or shape of graphics on the package (Fang \& Mowen, 2005; Westerman et al., 2012; Zhang et al., 2006); and (c) general settings (Banaei et al., 2017; Dazkir \& Read, 2012; Hess, Gryc, \& Hareli, 2013; Leder \& Carbon, 2005; Van Oel \& Van den Berkhof, 2013; Vartanian et al., 2013, 2017).

$\underline{\text { Items }}$

Regarding the first group of studies, Ghoshal et al. (2016) asked participants to rate the hedonic value and functionality of a selection of 30 common products. They rated higher hedonic perceptions for curved products and higher functional perceptions for angular products. The impact of curvature or angularity was moderated by individual differences such as need for cognition and involvement with the category.

Soranzo et al. (2018) created 3D physical artefacts that combined four characteristics: contour (curved vs. sharp), size (small vs. large), surface texture (rough vs. smooth), 
and behavior of the objects (lighting, sounding, vibrating, and quiescent). Participants had to interact with the object and rate it on each of seven dimensions: interesting, comfortable, playful, surprising, pleasant, special, and relaxing. For all dimensions except surprising, the curvature effect was significant. That is, curved objects were more interesting, more comfortable, more playful, more special, and more relaxing.

Another two studies focused on specific organic items: flowers (Hůla \& Flegr, 2016) and leaves (Hareli et al., 2016). Hůla and Flegr (2016) determined which, if any, flower colors and shapes were more preferred than others. The set of flower stimuli consisted of 52 photographs, which varied in different features: symmetry, form contour, color, complexity, and prototypicality. Twenty-one of them were classified as round, 15 as sharp, and 16 as mixed. According to their analyses, sharp contours positively affected the flower beauty scores, whereas mixed contours had no effect. However, we have to consider that the different features were not combined systematically. On another hand, the authors indicated the possibility that the curvature effect could be context-specific and, for some unspecified reason, does not apply to flowers. Despite its relative robustness, the curvature effect remains sensitive to methodological variations.

Hareli et al. (2016) asked participants to evaluate leaves -sharp-angled or curved- on a set of semantic differential scales. Curved shaped leaves were rated as more beautiful, friendlier, more comforting, and warmer than the sharp shaped ones. However, they also found a consistent pattern in which houses surrounded by sharp leafed vegetation were evaluated as more expensive, compared with houses surrounded by round leafed vegetation. In another experiment, they found that sharp leaf vegetation houses were evaluated as safer. They inferred that the characteristics associated with sharp shapes 
confer protection to the house. This way, we have to bear in mind that aesthetic preference is not the only way people choose between objects with different shapes.

\section{Product packaging and brand logos}

Fang and Mowen (2005) showed that product category moderated the effects of curvature on evaluations of brand logos. Specifically, participants preferred a round logo for vases but an angular logo for buildings. They proposed a match-up explanation to account for this finding. That is, when a logo matches the typical form of an object that is stored in consumers' mind, their evaluation tends to be more positive. However, Westerman et al. (2012) found no beneficial effect of semantic congruence associated with using angular and round shapes, demonstrating preference for curved designs for different types of products (chocolate, water, and bleach) and different packaging designs (box vs. bottle). They manipulated images of product packaging regarding the shape of both contour and graphics. There was a preference for curved designs that extended to self-report purchase likelihood. They also showed additive effects of contour and graphic shape that could not be accounted for by design typicality or perceived ease of use.

Also, Jiang et al. (2016) showed that the curved shape of a logo influenced consumers' inferences about products and their attitudes toward them. They showed that a curved brand logo activated mental associations related to "softness" and a sharp-angled brand logo activated mental associations related to "hardness," and these activated associations subsequently influenced product and company attribute judgments. The authors proposed mental imagery as the underlying mechanism driving the effect of logo shape on consumer judgments. Support was obtained from two experiments: One showing that the logo shape effects were eliminated under conditions of visual load but 
not cognitive load -because only the former type of load constrained the available resources in visuospatial working memory necessary for imagery generation-; a second experiment showed that the shape effect was reduced when people had a lower predisposition to generate mental images.

\section{$\underline{\text { General settings }}$}

Several studies examined how variation in the contour of interior spaces impacts explicit responses such as pleasure, aesthetics judgments, choices, and approach decisions (Banaei et al., 2017; Hess et al., 2013; Liu, Bogicevic, \& Mattila, 2018; Van Oel \& Van den Berkhof, 2013; Vartanian et al., 2013, 2017; Zhu \& Argo, 2013). In this line, Dazkir and Read (2012) tested pleasure and approach reactions toward curvilinear and rectilinear simulated interior settings. They focused specifically on furniture forms. The curvilinear forms elicited higher amounts of pleasant emotions (feeling relaxed, peaceful, calm) than the rectilinear forms. Also, the participants desired to approach curvilinear settings more compared with the rectilinear ones.

Van Oel and Van den Berkhof (2013) investigated the way passengers value architectural airport design characteristics, using visualizations of hypothetical areas. They used eight design characteristics with two levels each. The "form" characteristic was elaborated in the roof construction, as either a curvilinear or an orthogonal roof. The results showed that form was the most influential characteristic in choosing the most preferred passenger area. The curvilinear form was more preferred than the orthogonal form. The "layout" characteristic was the third most influential characteristic. The layout was elaborated as a straight area layout or a curved area 
layout. In general, passengers had a clear preference for an area with curved hallways.

In Vartanian et al. (2013), naive participants were more likely to judge spaces as beautiful if they had curvilinear rather than rectilinear contours. In contrast, contour had no effect on approach-avoidance decisions. In a second article, Vartanian et al. (2017) found that experts assessed curvilinear spaces as more beautiful than rectilinear spaces, whereas contour had no effect on beauty judgments among non-experts. Interestingly, when making approach-avoidance decisions, non-experts were more likely to choose to enter curvilinear rather than rectilinear spaces, whereas contour had no effect on approach-avoidance decisions among experts. Possible explanations of these results have been presented in the previous section about individual differences.

When contemplating architectural interiors as beautiful, Vartanian et al. (2013) found that curvilinear contour activated the anterior cingulate cortex (ACC) exclusively, a region strongly responsive to the reward properties and emotional salience of objects. The ACC also showed pronounced activity when participants in Banaei et al. (2017) presented higher responses in terms of pleasure and arousal related to rooms with more curved geometries. These authors investigated human brain dynamics connected to the affective impact of interior forms when the perceiver actively explored an architectural space. They used a mobile EEG setup synchronized to head-mounted virtual reality. Rooms associated with lower pleasure and arousal ratings contained more linear geometries, whereas rooms with higher pleasure and arousal ratings contained more curved geometries.

Other studies investigated the indirect influence of shapes on social attitudes and 
decisions (Hess et al., 2013; Liu et al., 2018; Zhu \& Argo, 2013). Hess et al. (2013) indicated that participants who were exposed to sharp shapes perceived other participants as more aggressive and were more likely to make an aggressive decision. In Experiment 1, participants assembled a puzzle. It was a face photo cut into round or sharp edged puzzle pieces. After completion of the puzzle, they rated the person whose photo they had assembled. When the photo was assembled from round shapes, participants rated the individual on the photo as significantly less aggressive than when the photo was assembled from sharp shapes. In Experiment 2, participants played a game and were told that their decisions would be paired with the decisions made by another unknown participant at a later time. The room was decorated with round or sharp objects of different materials. When the room was decorated with sharp objects, participants chose the aggressive option relatively more often than when the room was decorated with round shapes.

Zhu and Argo (2013) found that when seating arrangements were circular, consumers evaluated persuasive material more favorably when it was consistent with a belongingness need (i.e., it included family-oriented information or a majority endorsement). In contrast, when a seating arrangement contained angles, consumers preferred a persuasive message when it related to a uniqueness need (i.e., it included self-oriented information or a minority endorsement).

Liu et al. (2018) examined the impact of shape cues -curved versus sharp-angled- on customer satisfaction. The findings suggested that in non-busy settings, curved shape cue enhanced customer satisfaction. In contrast, sharp-angled shape cues increased customer satisfaction in busy settings. Customer satisfaction enhancement in non-busy settings was via warmth perceptions, and satisfaction enhancement in busy settings was 
through competence perceptions. The authors suggested that a service incorporating curved cues will activate customers' warmth associations, and one with sharp-angled cues will activate competence associations.

\section{Major Challenges, Goals, and Suggestions}

In the last section, we have seen that some studies about the curvature effect have been related to applied research in the last decade. It has been related to innovative sensory marketing strategies (Fang \& Mowen, 2005; Ghoshal et al., 2016; Liu et al., 2018; Schweinsberg et al., 2016; Zhang et al., 2006), social cognition (Hess et al., 2013; Zhu \& Argo, 2013), architecture (Banaei et al., 2017; Van Oel \& Van den Berkhof, 2013; Vartanian et al., 2013), security perception (Hareli et al., 2016), interior design (Dazkir \& Read, 2012), and car design (Carbon, 2010; Leder \& Carbon, 2005). We think this trend will continue in quite a few applied disciplines and is a great opportunity for researchers from empirical aesthetics. Interestingly, the effect has not yet been studied in the fields of painting, sculpture, and music, although some studies have shown its influence in dance and ballet (Aronoff, 2006; Aronoff et al., 1992; Christensen et al., 2016).

From another perspective, a comprehensive understanding of preference for curvature must go beyond the mere accumulation of data. According to Gómez-Puerto et al. (2016), research about the curvature effect needs to form some foundations of a unified framework to advance its understanding. For this purpose, we consider that there are some points that require research and refinement. Some of them are:

1. A conceptual approach. The concepts of curvature and curved are widely used 
throughout most published research. However, roundness and round are also used in some of the articles. It seems appropriate to clarify whether they refer to the same feature or whether there is some specific difference between the concepts. If there is consensus that they are referring to the same meaning, it may be more adequate to use only one pair of concepts to strengthen the field and avoid misunderstanding. Something similar is observed with sharpness and angularity. Gomez-Puerto et al. (2016) proposed the use of the dichotomy curvature/sharpness to describe the object of study, and curved/sharp-angled to characterize the stimuli causing the effect. Defining central concepts clearly and univocally should be the first step to build compelling and testable explanations of preference for curvature.

2. A psychophysical project. A disentanglement of the psychophysical nature of this feature is needed. We should know more about what is deemed to be curved, and what physical factors (number of vertices, concavity, size, complexity, and others) affect perceived curvature. Perhaps there are other nonphysical factors that can also affect it. For instance, Carbon (2010) mentioned the images of shark teeth, the outline of a shark, and a rose thorn as paradigmatical examples of sharp transitions in nature, but it could be argued that these stimuli are actually curved in contour, not sharp-angled. The psychophysical nature of the feature needs to be explored in order to determine which contours and lines people consider curved and which sharp-angled.

3. Preference for curvature, avoidance of sharpness, or both? Evidence for a genuine preference for curvature has been increasing in the last years, but there is also some evidence supporting the threat hypothesis. There is also a possibility that we are dealing with two cognitive phenomena or maybe only 
with one. When proposing an evolutionary origin, it is not enough to suggest a plausible explanation. A detailed scenario with testable predictions is required for both hypotheses to be useful.

4. Contextual and individual differences. Situations and personal characteristics can affect perceived curvature and preference of the contour. Previously, we have seen some of these characteristics that influence the curvature effect: selfconstrual, country, expertise, and openness to experience. However, we need some systematic studies that also help us to know more about the processes that moderate and mediate the curvature effect.

5. One or several preference mechanisms? We need to clarify whether we are dealing with a general preference for curvature or with specific dissociable preferences for curvatures. Is the mechanism that gives rise to preference for curved lines the same that gives rise to preference for curved contours? We can extend this question to $3 \mathrm{D}$ objects and also to different sensorial attributes and modalities. It is unclear whether this is a unimodal or a multimodal phenomenon.

Finally, we would like to include a suggestion for researchers, especially for beginners in research. Most of the studies used experimental designs to determine whether the effect is present in a particular situation, but it is appropriate to introduce manipulations of the participants' state and situations in order to reveal when the effect is present or when it increases or decreases in magnitude. This can be done using different experimental paradigms -implicit measures, eye movements, neuroimaging, among others- and would inform us more about the underlying cognitive processes that give rise to the preference for curvature. 


\section{References}

Aiken, N. E. (1998). The biological origins of art. Westport: Praeger Publishers/Greenwood.

Allen, G. (1877). Physiological aesthetics. New York, NY: D. Appleton \& Company.

Álvarez, A., Blanco, M., \& Leirós, L. (2002). Influencia de la simetría y la curvilinealidad en el procesamiento de estímulos cerrados. Psicothema, 14(3), $597-604$.

Amir, O., Biederman, I., \& Hayworth, K. J. (2011). The neural basis for shape preferences. Vision Research, 51(20), 2198-2206. https://doi.org/10.1016/j.visres.2011.08.015

Aronoff, J. (2006). How we recognize angry and happy emotion in people, places, and things. Cross-Cultural Research, 40(1), 83-105. https://doi.org/10.1177/1069397105282597

Aronoff, J., Woike, B. A., \& Hyman, L. M. (1992). Which are the stimuli in facial displays of anger and happiness: Configurational bases of emotion recognition. Journal of Personality and Social Psychology, 62(6), 1050.

https://doi.org/10.1037/0022-3514.62.6.1050

Banaei, M., Hatami, J., Yazdanfar, A., \& Gramann, K. (2017). Walking through architectural spaces: The impact of interior forms on human brain dynamics. Frontiers in Human Neuroscience, 11(September), 477. https://doi.org/10.3389/FNHUM.2017.00477

Bar, M., \& Neta, M. (2006). Humans prefer curved visual objects. Psychological Science, 17(8), 645-648. https://doi.org/10.1111/j.1467-9280.2006.01759.x Bar, M., \& Neta, M. (2007). Visual elements of subjective preference modulate 
amygdala activation. Neuropsychologia, 45(10), 2191-2200.

https://doi.org/10.1016/j.neuropsychologia.2007.03.008

Bertamini, M., \& Palumbo, L. (2015). The aesthetics of smooth contour curvature in historical contest. In Art and its role in the history of the Balkans. Kosovska, Mitrovica: Faculty of Philosophy, University of Pristina. Retrieved from https://www.bertamini.org/lab/Publications/BertaminiPalumbo2015.pdf

Bertamini, M., Palumbo, L., Gheorghes, T. N., \& Galatsidas, M. (2016). Do observers like curvature or do they dislike angularity? British Journal of Psychology, 107(1), 154-178. https://doi.org/10.1111/bjop.12132

Blazhenkova, O., \& Kumar, M. M. (2018). Angular versus curved shapes: Correspondences and emotional processing. Perception, 47(1), 67-89. https://doi.org/10.1177/0301006617731048

Carbon, C.C. (2010). The cycle of preference: Long-term dynamics of aesthetic appreciation. Acta Psychologica, 134(2), 233-244. https://doi.org/10.1016/j.actpsy.2010.02.004

Christensen, J. F., Pollick, F. E., Lambrechts, A., \& Gomila, A. (2016). Affective responses to dance. Acta Psychologica, 168, 91-105. https://doi.org/10.1016/j.actpsy.2016.03.008

Corradi, G. B., Rosselló, J., Vañó, J., Chuquichambi, E., Bertamini, M., \& Munar, E. (2018). The effects of presentation time on preference for curvature of real objects and meaningless novel patterns. British Journal of Psychology. https://doi.org/DOI:10.1111/bjop.12367

Cotter, K. N., Silvia, P. J., Bertamini, M., Palumbo, L., \& Vartanian, O. (2017). Curve appeal: Exploring individual differences in preference for curved versus angular objects. I-Perception, 8(2), 1-17. https://doi.org/10.1177/2041669517693023 
Dazkir, S. S., \& Read, M. A. (2012). Furniture form and their influence on our emotional responses toward interior environments. Environment and Behavior, 44(5), 722-732. https://doi.org/10.1177/0013916511402063

Dewey, J. (1922). Human nature and conduct. Carbondale, IL: Southern Illinois University Press.

Elliot, A. J., \& Covington, M. V. (2001). Approach and avoidance motivation. Educational Psychology Review, 13(2), 73-92. https://doi.org/10.1023/A:1009009018235

Fang, X., \& Mowen, J. C. (2005). Exploring factors influencing logo effectiveness. An experimental inquiry. Advances in Consumer Research, 32, 161.

Fantz, R. L. (1961). The origin of form perception. Scientific American, 204(5), 66-72. https://doi.org/10.1038/scientificamerican0561-66

Fantz, R. L., \& Miranda, S. B. (1975). Newborn-infant attention to form of contour. Child Development, 46(1), 224-228. https://doi.org/10.2307/1128853

Franck, K., \& Rosen, E. (1949). A projective test of masculinity-femininity. Journal of Consulting Psychology, 13(4), 247-256.

Ghoshal, T., Boarwright, P., \& Malika, M. (2016). Curvature for all angles. In The Psychology of Design (pp. 91-106). New York: Routeledge.

Gomez-Puerto, G., Rosselló, J., Corradi, G., Acedo-Carmona, C., Munar, E., \& Nadal, M. (2017). Preference for curved contours across cultures. Psychology of Aesthetics, Creativity, and the Arts, 12(4), 432-439.

https://doi.org/10.1037/aca0000135

Gomez-Puerto, G., Munar, E., \& Nadal, M. (2016). Preference for curvature: A historical and conceptual framework. Frontiers in Human Neuroscience, 9(January), 1-8. https://doi.org/10.3389/fnhum.2015.00712 
Grassini, S., Railo, H., Valli, K., Revonsuo, A., \& Koivisto, M. (2019). Visual features and perceptual context modulate attention towards evolutionarily relevant threatening stimuli: Electrophysiological evidence. Emotion, 19(2), 348-364. https://doi.org/10.1037/emo0000434

Guthrie, G., \& Wiener, M. (1966). Subliminal perception or perception of partial cue with pictorial stimuli. Journal of Personality and Social Psychology, 3(6), 619628. https://doi.org/10.1037/h0023197

Hareli, S., David, S., Lev-Yadun, S., \& Katzir, G. (2016). Money in your palm: Sharp shaped vegetation in the surroundings increase the subjective value of houses. Journal of Environmental Psychology, 46, 176-187. https://doi.org/10.1016/j.jenvp.2016.04.014

Hess, U., Gryc, O., \& Hareli, S. (2013). How shapes influence social judgments. Social Cognition, 31(1), 72-80. https://doi.org/10.1521/soco.2013.31.1.72

Hevner, K. (1935). Experimental studies of the affective value of colors and lines. Journal of Applied Psychology, 19(4), 385-398.

Hogarth, W. (1753). The analysis of beauty: Written with a view of fixing the fluctuating ideas of taste. Ann Arbor, MI: University of Michigan Library.

Hopkins, J. R., Kagan, J., Brachfeld, S., Hans, S., \& Linn, S. (1976). Infant responsivity to curvature. Child Development, 47(4), 1166-1171.

Hůla, M., \& Flegr, J. (2016). What flowers do we like? The influence of shape and color on the rating of flower beauty. PeerJ, 4:e2106; $D$. https://doi.org/10.7717/peerj.2106

Jadva, V., Hines, M., \& Golombok, S. (2010). Infants' preferences for toys, colors, and shapes: Sex differences and similarities. Archives of Sexual Behavior, 39(6), 12611273. https://doi.org/10.1007/s10508-010-9618-z 
Jahoda, G. (1956). Sex differences in preferences for shapes: A cross-cultural replication. British Journal of Psychology, 47(2), 126-132.

Jakesch, M., \& Carbon, C.-C. (2011). Humans prefer curved objects on basis of haptic evaluation. Perception, 40(Supplement), 219. https://doi.org/10.1068/v110148

Jiang, Y., Gorn, G. J., Galli, M., \& Chattopadhyay, A. (2016). Does your company have the right logo? How and why circular- and angular-logo shapes influence brand attribute judgments. Journal of Consumer Research, 42(5), 709-726. https://doi.org/10.1093/jcr/ucv049

Kastl, A. J., \& Child, I. L. (1968). Emotional meaning of four typographical variables. Journal of Applied Psychology, 52(6, Pt.1), 440-446. https://doi.org/10.1037/h0026506

Krout, J. (1950). Symbol elaboration test (S.E.T.): The reliability and validity a new projective technique. Psychological Monographs: General and Applied, 64(4), 167.

Leder, H., \& Carbon, C.-C. (2005). Dimensions in appreciation of car interior design. Applied Cognitive Psychology, 19(5), 603-618. https://doi.org/10.1002/acp.1088

Leder, H., Carbon, C.-C., \& Ripsas, A.-L. (2006). Entitling art: Influence of title information on understanding and appreciation of paintings. Acta Psychologica, 121(2), 176-198. https://doi.org/10.1016/j.actpsy.2005.08.005

Leder, H., Tinio, P. P. L., \& Bar, M. (2011). Emotional valence modulates the preference for curved objects. Perception, 40(6), 649-655. https://doi.org/10.1068/p6845

Liu, S. Q., Bogicevic, V., \& Mattila, A. S. (2018). Circular vs. angular servicescape: "Shaping" customer response to a fast service encounter pace. Journal of Business Research, 89(April), 47-56. https://doi.org/10.1016/j.jbusres.2018.04.007 
LoBue, V. (2014). Deconstructing the snake: The relative roles of perception, cognition, and emotion on threat detection. Emotion, 14(4), 701-711. https://doi.org/10.1037/a0035898

Lundholm, H. (1920). The affective tone of lines: Experimental researches. Psychological Review, 28(1), 43-60.

Marr, D. (1982). Vision. A Computational Investigation into the Human Representation and Processing of Visual Information. Cambridge, MA: MIT Press. https://doi.org/https://doi.org/10.7551/mitpress/9780262514620.001.0001

McElroy, W. A. (1954). A sex difference in preferences for shapes. British Journal of Psychology, 64(4), 209-216.

Munar, E., Gómez-Puerto, G., Call, J., \& Nadal, M. (2015). Common visual preference for curved contours in humans and great apes. Plos One, 10 (11): e0141106 https://doi.org/10.1371/journal.pone.0141106

Munar, E., Gómez-Puerto, G., \& Gomila, A. (2014). The evolutionary roots of aesthetics: An approach-avoidance look at curvature preference. In A. Scarinzi (Ed.), Embodied Aesthetics (pp. 3-17). Leiden: Brill.

Munroe, R. H., Munroe, R. L., \& Lansky, L. M. (1976). A sex difference in shape preference. The Journal of Social Psychology, 98, 139-140.

Palumbo, L., \& Bertamini, M. (2016). The curvature effect: A comparison between preference tasks. Empirical Studies of the Arts, 34(1), 35-53. https://doi.org/10.1177/0276237415621185

Palumbo, L., Ruta, N., \& Bertamini, M. (2015). Comparing angular and curved shapes in terms of implicit associations and approach/avoidance responses. Plos One, 10(10), e0140043. https://doi.org/10.1371/journal.pone.0140043

Poffenberger, A. T., \& Barrows, B. E. (1924). The feeling value of lines. Journal of 
Applied Psychology, 8(2), 187-205.

Ponce, C. R., Hartmann, T. S., \& Livingstone, M. S. (2017). End-stopping predicts curvature tuning along the ventral stream. The Journal of Neuroscience, 37(3), 648-659. https://doi.org/10.1523/JNEUROSCI.2507-16.2017

Quinn, P. C., Brown, C. R., \& Streppa, M. L. (1997). Perceptual organization of complex visual configurations by young infants. Infant Behavior and Development, 20(1), 35-46. https://doi.org/10.1016/S0163-6383(97)90059-X

Reber, R., Wurtz, P., \& Zimmermann, T. D. (2004). Exploring “fringe” consciousness: the subjective experience of perceptual fluency and its objective bases. Consciousness and Cognition, 13(1), 47-60. https://doi.org/10.1016/S10538100(03)00049-7

Ruff, H. A., \& Birch, H. G. (1974). Infant visual fixation: The effect of concentricity, curvilinearity and number of directions. Journal of Experimental Child Psychology, 17, 460-473.

Ruta, N., Palumbo, L., \& Bertamini, M. (2014). Comparing angular and smoothe polygons. Exploring the link between preference, response time and contour integration. Proceedings of the 23rd IAEA Conference, 571-575.

Salgado-Montejo, A., Alvarado, J. A., Velasco, C., Salgado, C. J., Hasse, K., \& Spence, C. (2015). The sweetest thing: The influence of angularity, symmetry, and the number of elements on shape-valence and shape-taste matches. Frontiers in Psychology, 6(April), 1-17. https://doi.org/10.3389/fpsyg.2015.01382

Schneirla, T. C. (1965). Aspects of stimulation and organization in approach/withdrawal processes underlying vertebrate behavioral development. Advances in the Study of Behavior, 1(C), 1-74. https://doi.org/10.1016/S0065-3454(08)60055-8

Schweinsberg, M., Madan, N., Vianello, M., Sommer, S. A., Jordan, J., Tierney, W., ... 
Uhlmann, E. L. (2016). The pipeline project: Pre-publication independent replications of a single laboratory's research pipeline. Journal of Experimental Social Psychology, 66, 55-67. https://doi.org/10.1016/j.jesp.2015.10.001

Silvia, P. J., \& Barona, C. M. (2009). Do people prefer curved objects? Angularity, expertise, and aesthetic preference. Empirical Studies of the Arts, 27(1), 25-42. https://doi.org/10.2190/EM.27.1.b

Soranzo, A., Petrelli, D., Ciolfi, L., \& Reidy, J. (2018). On the perceptual aesthetics of interactive objects. Quarterly Journal of Experimental Psychology, 174702181774922. https://doi.org/10.1177/1747021817749228

Stratton, G. (1902). Eye-movements and the aesthetics of visual form. Philosophische Studien, 20, 336-359.

Stratton, G. (1906). Symmetry, linear Illusions, and the movements of the eye. Psychological Review, 13(2), 82-96. https://doi.org/http://dx.doi.org/10.1037/h0072441

Treisman, A. M., \& Gelade, G. (1980). A feature-integration theory of attention. Cognitive Psychology, 12(1), 97-136.

Tzeng, O. C., Trung, N. T., \& Rieber, R. W. (1990). Cross-cultural comparisons of psychosemantics of icons and graphics. Internationa Journal of Psychology, 25(1), 77-97.

Uher, J. (1991). On zigzag designs: Three levels of meaning. Current Anthropology, 32(4), 437-439.

Valentine, C. (1950). Psychology and its bearing on education. Hove (UK): Routeledge.

Van Oel, C. J., \& Van den Berkhof, F. W. D. (2013). Consumer preferences in the design of airport passenger areas. Journal of Environmental Psychology, 36, 280- 
290. https://doi.org/10.1016/j.jenvp.2013.08.005

Vartanian, O., Navarrete, G., Chatterjee, A., Fich, L. B., Leder, H., Modrono, C., ... Skov, M. (2013). Impact of contour on aesthetic judgments and approachavoidance decisions in architecture. Proceedings of the National Academy of Sciences of the United States of America, 110, 10446-10453. https://doi.org/10.1073/pnas.1301227110

Vartanian, O., Navarrete, G., Chatterjee, A., Fich, L. B., Leder, H., Rostrup, N., ... Skov, M. (2017). Preference for curvilinear contour in interior architectural spaces: Evidence from experts and nonexperts preference. Psychology of Aesthetics, Creativity, and the Arts, 13(1), 110-116. https://doi.org/10.1037/aca0000150

Velasco, C., Salgado-Montejo, A., Elliot, A. J., Woods, A. T., Alvarado, J., \& Spence, C. (2016). The shapes associated with approach/avoidance words. Motivation and Emotion, 40(5), 689-702. https://doi.org/10.1007/s11031-016-9559-5

Velasco, C., Woods, A. T., Hyndman, S., \& Spence, C. (2015). The taste of typeface. IPerception, 6(4), 1-10.

Velasco, C., Woods, A. T., Petit, O., Cheok, A. D., \& Spence, C. (2016). Crossmodal correspondences between taste and shape, and their implications for product packaging: A review. Food Quality and Preference, 52(4), 17-26. https://doi.org/10.1016/j.foodqual.2016.03.005

Westerman, S., Gardner, P. H., Sutherland, E. J., White, T., Jordan, K., Watts, D., \& Wells, S. (2012). Product design: Preference for rounded versus angular design elements. Psychology \& Marketing, 29(August), 595-605. https://doi.org/10.1002/mar

Wolfe, J. M., Yee, A., \& Friedman-Hill, S. R. (1992). Curvature is a basic feature for visual search tasks. Perception, 21, 465-480. 
Yue, X., Pourladian, I. S., Tootell, R. B. H., \& Ungerleider, L. G. (2014). Curvatureprocessing network in macaque visual cortex. Proceedings of the National Academy of Sciences, 111(33), E3467-E3475.

https://doi.org/10.1073/pnas.1412616111

Zhang, Y., Feick, L., \& Price, L. J. (2006). The impact of self-construal on aesthetic preference for angular versus rounded shapes. Personality and Social Psychology Bulletin, 32(August), 794-805. https://doi.org/10.1177/0146167206286626

Zhu, R., \& Argo, J. J. (2013). Exploring the impact of various shaped seating arrangements on persuasion. Journal of Consumer Research, 40(2), 336-349. https://doi.org/10.1086/670392 